\title{
Ultra-Quasi-Metrically Tight Extensions of Ultra-Quasi-Metric Spaces
}

\author{
Collins Amburo Agyingi \\ African Institute for Mathematical Sciences, Crystal Gardens, P.O. Box 608, Limbe, Cameroon \\ Correspondence should be addressed to Collins Amburo Agyingi; agyingic@gmail.com
}

Received 2 July 2015; Accepted 16 August 2015

Academic Editor: Luoshan Xu

Copyright (C) 2015 Collins Amburo Agyingi. This is an open access article distributed under the Creative Commons Attribution License, which permits unrestricted use, distribution, and reproduction in any medium, provided the original work is properly cited.

The concept of the tight extension of a metric space was introduced and studied by Dress. It is known that Dress theory is equivalent to the theory of the injective hull of a metric space independently discussed by Isbell some years earlier. Dress showed in particular that for a metric space $X$ the tight extension $T_{X}$ is maximal among the tight extensions of $X$. In a previous work with $P$. Haihambo and H.-P. Künzi, we constructed the tight extension of a $T_{0}$-quasi-metric space. In this paper, we continue these investigations by presenting a similar construction in the category of $U Q P$-metric spaces and nonexpansive maps.

\section{Introduction}

In [1] a concept of tight extension that is appropriate in the category of $T_{0}$-quasi-metric spaces and nonexpansive maps was studied. In particular such an extension was constructed and it was shown that this extension is maximal among the tight extensions.

In this paper we will show how the studies of [1] can be modified in order to obtain a theory that is appropriate for $U Q P$-metric spaces. By UQP-metric space in the following, we mean $T_{0}$-ultra-quasi-metric spaces. Even though our studies follow essentially $[1,2]$, we found it imperative to work out every detail of this theory in this paper.

We will show that every UQP-metric space $X$ has a $u q$-tight extension which is maximal amongst the $u q$-tight extensions of $X$. This agrees with the result we have for $T_{0}$ quasi-metric spaces (check [1]).

\section{Preliminaries}

We mention that the ultra-quasi-pseudometric spaces should not be confused with the quasi-ultrametrics as they are discussed in the theory of dissimilarities (check, e.g., [3]).

Definition 1 (compare [4, page 2]). Let $X$ be a set and let $d: X \times X \rightarrow[0, \infty)$ be a mapping into the set $[0, \infty)$ of nonnegative reals. Then $d$ is an ultra-quasi-pseudometric or, for short, a uqp-metric on on $X$ if

(a) $d(x, x)=0$ for all $x \in X$,

(b) $d(x, z) \leq \max \{d(x, y), d(y, z)\}$ whenever $x, y, z \in X$.

We remark here that the conjugate $d^{t}$ of $d$ where $d^{t}(x, y)=d(y, x)$ whenever $x, y \in X$ is also uqp-metric on $X$.

If $d$ also satisfies the condition,

(c) for any $x, y \in X, d(x, y)=0=d(y, x)$ implies that $x=y$,

then $d$ is called $U Q P$-metric on $X$.

Notice that $d^{s}=\max \left\{d, d^{t}\right\}=d \vee d^{t}$ is ultrametric on $X$.

Example 2 (compare [4, Example 2]). Note that, for $X=$ $[0, \infty)$, the pair $(X, n)$ is UQP-metric space, where $n$ is such that $n(x, y)=x$ if $x, y \in X$ and $x>y$, and $n(x, y)=0$ if $x, y \in$ $X$ and $x \leq y$. We show the strong triangle inequality $n(x, z) \leq$ $\max \{n(x, y), n(y, z)\}$ whenever $x, y, z \in X$ since the other conditions are obvious. For $n(x, y)=x$, the result is trivial, since $n(x, z) \leq n(x, y)$. Similarly the case that $n(x, y)=0$ and $n(y, z)=y$ is obvious, since $x \leq y$ and $n(x, z) \leq n(y, z)$. In the remaining case that $n(x, y)=0=n(y, z)$, we have by transitivity of $\leq$ that $x \leq z$, and thus $n(x, z)=0$. It is obvious that $n$ satisfies the $T_{0}$-condition. 
Notice also that, for $x, y \in[0, \infty)$, we have $n^{s}(x, y)=$ $\max \{x, y\}$ if $x \neq y$ and $n^{s}(x, y)=0$ if $x=y$. The ultrametric $n^{s}$ is complete on $[0, \infty)$ since $n$ and $n^{-1}$ are bicomplete on $[0, \infty)$. Recall that a $U Q P$-metric space $(X, d)$ is said to be bicomplete if the ultrametric space $\left(X, d^{s}\right)$ is complete.

Furthermore 0 is the only nonisolated point of $\tau\left(n^{s}\right)$. Indeed $A=\{0\} \cup\{1 / n: n \in \mathbb{N}\}$ is a compact subspace of $\left([0, \infty), n^{s}\right)$.

In some cases we will replace $[0, \infty)$ with $[0, \infty]$ and, in this case, we will speak of an extended $u q p$-metric.

Lemma 3 (compare [5, Proposition 2.1]). Let $\alpha, \beta, \gamma \in$ $[0, \infty)$. Then the following are equivalent:
(a) $n(\alpha, \beta) \leq \gamma$,
(b) $\alpha \leq \max \{\beta, \gamma\}$.

Proof.

(a) $\Rightarrow(b)$. To reach a contradiction, suppose that $\alpha>$ $\max \{\beta, \gamma\}$. Since $\alpha>\beta$, we have $n(\alpha, \beta)=\alpha \leq \gamma$ by part (a) and the way $n$ was defined. Thus, we would get $\alpha \leq$ $\max \{\beta, \gamma\}<\alpha$, a contradiction.

$(b) \Rightarrow(a)$. Suppose that, on the contrary, $n(\alpha, \beta)>\gamma$ would hold. Then $\alpha>\beta$ and, hence, $\alpha>\gamma$ would hold which would imply $\alpha>\max \{\beta, \gamma\}$ in contradiction to our assumption $\alpha \leq$ $\max \{\beta, \gamma\}$.

The following corollaries are immediate. Their proofs rely on Lemma 3.

Corollary 4 (see $[4])$. Let $(X, d)$ be uqp-metric space. Consider a map $f: X \rightarrow[0, \infty)$ and let $x, y \in X$. Then the following are equivalent:

(a) $n(f(x), f(y)) \leq d(x, y)$,

(b) $f(x) \leq \max \{f(y), d(x, y)\}$.

Definition 5. A map $f:\left(X, d_{X}\right) \rightarrow\left(Y, d_{Y}\right)$ between two uqp-metric spaces $\left(X, d_{X}\right)$ and $\left(Y, d_{Y}\right)$ is called nonexpansive if $d_{Y}(f(x), f(y)) \leq d_{X}(x, y)$ holds for any $x, y \in X$.

Corollary 6 (see $[4])$. Let $(X, d)$ be uqp-metric space. Then

(a) $f:(X, d) \rightarrow([0, \infty), n)$ is a nonexpansive map if and only if $f_{2}(x) \leq \max \left\{f_{1}(y), d(x, y)\right\}$ holds for all $x, y \in X$,

(b) $f:(X, d) \rightarrow\left([0, \infty), n^{-1}\right)$ is a nonexpansive map if and only if $f_{1}(x) \leq \max \left\{f_{2}(y), d(y, x)\right\}$ holds for all $x, y \in X$.

Definition 7. A map $f:\left(X, d_{X}\right) \rightarrow\left(Y, d_{Y}\right)$ between two uqpmetric spaces $\left(X, d_{X}\right)$ and $\left(Y, d_{Y}\right)$ is said to be an isometry provided that $d_{Y}(f(x), f(y))=d_{X}(x, y)$ whenever $x, y \in$ $X$. Two uqp-metric spaces $\left(X, d_{X}\right)$ and $\left(Y, d_{Y}\right)$ are said to be isometric provided that there exists a bijective isometry between them. Note that if $\left(X, d_{X}\right)$ is a $U Q P$-metric space, then $f$ is injective.

\section{Ultra-Ample Function Pairs on $U Q P-M e t r i c$ Space}

We will recall some results from the theory of hyperconvex hulls of $U Q P$-metric spaces due to [4].

Definition 8 (compare [4, Definition 1, page 4]). Let $(X, d)$ be $U Q P$-metric space. One will say that a function pair $f=$ $\left(f_{1}, f_{2}\right)$ on $(X, d)$ where $f_{i}: X \rightarrow[0, \infty)(i=1,2)$ is ultraample if, for all $x, y \in X$, one has $d(x, y) \leq \max \left\{f_{2}(x), f_{1}(y)\right\}$.

Let us denote by $u P_{X}$ the set of all ultra-ample function pairs on $U Q P$-metric space $(X, d)$. For each $f, g \in u P_{X}$, define

$$
\begin{gathered}
N(f, g)=\max \left\{\sup _{x \in X} n\left(f_{1}(x), g_{1}(x)\right),\right. \\
\left.\sup _{x \in X} n\left(g_{2}(x), f_{2}(x)\right)\right\},
\end{gathered}
$$

where the UQP-metric (of course we can use UQP-metric $n$ here since the function pairs take values in $[0, \infty)) n$ is as defined in Example 2. Then $N$ is an extended $U Q P$-metric on $u P_{X}$.

Lemma 9. Let $(X, d)$ be UQP-metric space and let $a \in X$. Then one has that $f_{a}(x):=(d(a, x), d(x, a))$ whenever $x \in X$ is an ultra-ample function pair belonging to $u P_{X}$.

We say that a function pair $f$ is uq-minimal among the ultra-ample function pairs on $(X, d)$ if it is an ultra-ample function pair and if $g=\left(g_{1}, g_{2}\right)$ is an ultra-ample function pair on $(X, d)$ and for each $x \in X g_{1}(x) \leq f_{1}(x)$ and $g_{2}(x) \leq$ $f_{2}(x)$, which implies $f=g$. We will also call uq-minimal ultraample function pair uq-extremal (ultra-ample) function pair. By $u Q_{X}$ we will denote the set of all uq-extremal function pairs on $(X, d)$ equipped with the restriction of $N$ to $u Q_{X}$, which we will still denote by N. Of course $N$ is actually UQP-metric on $u Q_{X}$ (compare Corollary 5 of [4]). We will call $\left(u Q_{X}, N\right)$ the ultra-quasi-metrically injective hull of $(X, d)$.

Lemma 10. Let $(X, d)$ be UQP-metric space and let $f \in u Q_{X}$. For all $x, y \in X,\left(f_{1}(x)>f_{1}(y)\right)$ implies that $f_{1}(x) \leq d(y, x)$ and $\left(f_{2}(x)>f_{2}(y)\right)$ implies that $f_{2}(x) \leq d(x, y)$.

Proof. See the proof of Lemma 3 of [4].

As a corollary, we have the following.

Corollary 11. Let $(X, d)$ be UQP-metric space. If $f$ is a minimal ultra-ample function pair on $(X, d)$, then $f_{1}(x) \leq$ $\max \left\{f_{1}(y), d(y, x)\right\}$ and $f_{2}(x) \leq \max \left\{f_{2}(y), d(x, y)\right\}$ whenever $x, y \in X$. Thus the maps $f_{1}:(X, d) \rightarrow\left([0, \infty), n^{-1}\right)$ and $f_{2}:(X, d) \rightarrow([0, \infty), n)$ are contracting maps (check, e.g., Corollary 6).

Lemma 12. Let $f$ be a minimal ultra-ample function pair on a $T_{0}$-ultra-quasi-metric space $(X, d)$. Then

$$
\begin{aligned}
f_{1}(x) & =\sup \left\{d(y, x): y \in X, d(y, x)>f_{2}(y)\right\} \\
& =\sup \left\{\left(f_{x}\right)_{2}(y): y \in X,\left(f_{x}\right)_{2}(y)>f_{2}(y)\right\},
\end{aligned}
$$




$$
\begin{aligned}
f_{2}(x) & =\sup \left\{d(x, y): y \in X, d(x, y)>f_{1}(y)\right\} \\
& =\sup \left\{\left(f_{x}\right)_{1}(y): y \in X,\left(f_{x}\right)_{1}(y)>f_{1}(y)\right\}
\end{aligned}
$$

whenever $x \in X$.

Proof. See the proof of Lemma 4 of [4].

The following lemma and its proof are found in [4].

Lemma 13. If $f$ and $g$ are minimal ultra-ample function pairs on a UQP-metric space $(X, d)$, then

$$
\begin{aligned}
N(f, g) & =\sup _{x \in X} n\left(f_{1}(x), g_{1}(x)\right) \\
& =\sup _{x \in X} n\left(g_{2}(x), f_{2}(x)\right) .
\end{aligned}
$$

As a consequence of Lemmas 12 and 13 we have the following corollary.

Corollary 14. Any minimal ultra-ample function pair $f=$ $\left(f_{1}, f_{2}\right)$ on UQP-metric space $(X, d)$ satisfies the following:

$$
\begin{aligned}
& f_{1}(x)=\sup \left\{n\left(d(y, x), f_{2}(y)\right): y \in X\right\}, \\
& f_{2}(x)=\sup \left\{n\left(d(x, y), f_{1}(y)\right): y \in X\right\}
\end{aligned}
$$

whenever $x \in X$.

\section{UQP-Metric Tight Extensions}

In this paper we will study uq-tight extensions as defined below in Definition 19. Moreover, by uq-tight extensions, we will mean tight extensions of $U Q P$-metric spaces.

Proposition 15 (compare [2, Section 1.3]). Let $(X, d)$ be $U Q P$-metric space. Then $u Q_{X}$ consists of all functions pairs which are "minimal" in $u P_{X}$.

Proof. To prove this proposition, we prove that there is no $g \in$ $u P_{X}$ with $g<f$ but $g \neq f$. This is so since, on the one hand, $g \leq f \in u Q_{X}$ and $g \in U P_{X}$ imply

$$
\begin{aligned}
& f_{2}(x) \\
& \quad=\sup \left\{d(x, y): y \in X, d(x, y)>f_{1}(y) \geq g_{1}(y)\right\} \\
& \quad=\sup \{d(x, y): y \in X, d(x, y)>g(y)\} \leq g_{2}(x) .
\end{aligned}
$$

Thus

$$
f_{2}(x) \leq g_{2}(x) .
$$

Using (6) and the condition that $g_{2} \leq f_{2}$, we have that $f_{2}=g_{2}$.

In the same manner we can show that $f_{1}=g_{1}$ so as to conclude that $f=g$.

On the other hand, suppose that, for some $x \in X$ and $f \in$ $u P_{X}$, we have that $f_{2}(x)>\sup \{d(x, y): y \in X$ and $d(x, y)>$ $\left.f_{1}(y)\right\}$.
For each $x \in X$ and $f \in u P_{X} \operatorname{set}\left(p_{x}(f)\right)_{1}(z)=f_{1}(z)$ if $z \epsilon$ $X \backslash\{x\}$ and $\left(p_{x}(f)\right)_{1}(x)=\sup \{d(y, x): y \in X$ and $d(y, x)>$ $\left.f_{2}(y)\right\}$.

Similarly for each $x \in X$ and $f \in u P_{X}$ set $\left(p_{x}(f)\right)_{2}(z)=$ $f_{2}(z)$ if $z \in X \backslash\{x\}$ and $\left(p_{x}(f)\right)_{2}(x)=\sup \{d(x, y): y \in$ $X$ and $\left.d(x, y)>f_{1}(y)\right\}$.

We show first that $p_{x}(f)$ is ultra-ample. We will consider the following cases.

Case 1. If $z=x$ and $y=x$, then the result holds since $d(x, x)=$ 0 .

Case 2. If $z \neq x$ and $y \neq x$, then $\left(p_{x}(f)\right)_{2}(z)=f_{2}(z)$, so that

$$
\begin{aligned}
\max & \left\{\left(p_{x}(f)\right)_{2}(z),\left(p_{x}(f)\right)_{1}(y)\right\} \\
& =\max \left\{f_{2}(z), f_{1}(y)\right\} \geq d(z, y) .
\end{aligned}
$$

Case 3. Consider $z=x$ and $y \neq x$. In this case $\left(p_{x}(f)\right)_{1}(y)=$ $f_{1}(y)$ and $\left(p_{x}(f)\right)_{2}(z)=\sup \{d(z, y): y \in X$ and $d(z, y)>$ $\left.f_{1}(y)\right\}$, so that

$$
\begin{aligned}
& \max \left\{\left(p_{x}(f)\right)_{2}(z),\left(\left(p_{x}\right)(f)\right)_{1}(y)\right\} \\
& \quad=\max \left\{\sup \left\{d(z, y): y \in X, d(z, y)>f_{1}(y)\right\},\right. \\
& \left.f_{1}(y)\right\} \geq d(z, y) .
\end{aligned}
$$

Case 4. In a manner similar to case 3 , the result can be shown.

Thus $p_{x}(f)$ is ultra-ample and also satisfies $p_{x}(f) \leq f$ by the way it was constructed.

Thus by taking $g=p_{x}(f)$, we can conclude that, for any $f \in u P_{X}, g \leq f$.

Lemma 16 (compare [4, Lemma 8]). Let $(X, d)$ be UQPmetric space. Then, for any $f, g \in u Q_{X}$, we have that

$$
\begin{aligned}
& N(f, g)=\sup \left\{d\left(x_{1}, x_{2}\right): x_{1}, x_{2} \in X, d\left(x_{1}, x_{2}\right)\right. \\
& \left.\quad>f_{2}\left(x_{1}\right), d\left(x_{1}, x_{2}\right)>g_{1}\left(x_{2}\right)\right\} .
\end{aligned}
$$

Proposition 17 (compare [1, Proposition 1]). Let $(X, d)$ be $U Q P-$ metric space. There exists a retraction map $p: u P_{X} \rightarrow$ $u Q_{X}$, that is, a map that satisfies the following conditions:

(a) $N(p(f), p(g)) \leq N(f, g)$ whenever $f, g \in u P_{X}$.

(b) $p(f) \leq f$ whenever $f \in u P_{X}$.

(In particular we have that $p(f)=f$ whenever $f \in$ $\left.u Q_{X} \cdot\right)$

Proof. We will prove Proposition 17 by the use of Zorn's lemma.

Indeed, let $(X, d)$ be $U Q P$-metric space and let $\mathscr{P}$ be the set of all maps from $u P_{X}$ to $u P_{X}$ satisfying conditions (a) and (b) in Proposition 17.

Order $\mathscr{P}$ by

$$
\begin{aligned}
p & \leq q \\
& \Longleftrightarrow p(f) \leq q(f), \\
& \Longleftrightarrow N(p(f), p(g)) \leq N(q(f), q(g))
\end{aligned}
$$


for all $f, g \in u P_{X}$ and $p, q \in \mathscr{P}$. Then $\mathscr{P} \neq \emptyset$ since the identity map belongs to $\mathscr{P}$.

We have to check now that $\preceq$ is actually a partial order.

Reflexivity is obvious since every map is equal to itself.

Let now $p, q \in \mathscr{P}$ such that $p \preceq q$ and $q \preceq p$. Consider

$$
\begin{aligned}
p & \leq q \\
& \Longrightarrow(p(f))_{1} \leq(q(f))_{1} \\
& \Longrightarrow(p(f))_{2} \leq(q(f))_{2}, \\
& \Longrightarrow N(p(f), p(g)) \leq N(q(f), q(g))
\end{aligned}
$$

$$
q \preceq p
$$

$$
\begin{aligned}
& \Longrightarrow(q(f))_{1} \leq(p(f))_{1}, \\
& \Longrightarrow(q(f))_{2} \leq(p(f))_{2}, \\
& \Longrightarrow N(q(f), q(g)) \leq N(p(f), p(g)) .
\end{aligned}
$$

$(p(f))_{1} \leq(q(f))_{1}$ and $(q(f))_{1} \leq(p(f))_{1}$ imply that $(p(f))_{1}=$ $(q(f))_{1}$. In a similar manner, we have that $(p(f))_{2}=(q(f))_{2}$ so that we can conclude that $p=q$.

Also $N(p(f), p(g)) \leq N(q(f), q(g))$ and $N(q(f), q(g)) \leq$ $N(p(f), p(g))$ imply that $p=q$. This shows that $\preceq$ is antisymmetric.

Suppose now that $p, q, s \in \mathscr{P}$ such that $p \preceq q$ and $q \preceq s$ :

$$
\begin{aligned}
p & \leq q \\
& \Longrightarrow(p(f))_{1} \leq(q(f))_{1}, \\
& \Longrightarrow(p(f))_{2} \leq(q(f))_{2}, \\
& \Longrightarrow N(p(f), p(g)) \leq N(q(f), q(g)) \\
q & \preceq s \\
& \Longrightarrow(q(f))_{1} \leq(s(f))_{1}, \\
& \Longrightarrow(q(f))_{2} \leq(s(f))_{2}, \\
& \Longrightarrow N(q(f), q(g)) \leq N(s(f), s(g)) .
\end{aligned}
$$

$(p(f))_{1} \leq(q(f))_{1}$ and $(q(f))_{1} \leq(s(f))_{1}$ imply that $(p(f))_{1} \leq$ $(s(f))_{1}$ by transitivity of $[0, \infty)$ as a subset of $\mathbb{R}$ with the usual ordering $\leq$. Similarly, we can show that $(p(f))_{2} \leq(s(f))_{2}$.

Also $N(p(f), p(g)) \leq N(q(f), q(g))$ and $N(q(f), q(g)) \leq$ $N(s(f), s(g))$ imply that $N(p(f), p(g)) \leq N(s(f), s(g))$. Thus $p \preceq s$. This proves that $\preceq$ is transitive. Therefore $(\mathscr{P}, \preceq)$ is a partially ordered set.

Next we show that every chain in $\mathscr{P}$ has a lower bound.

Let $\emptyset \neq \mathscr{K} \subseteq \mathscr{P}$ be a chain and define $s: u P_{X} \rightarrow u P_{X}$ by

$$
s(f)(x):=\left(\inf _{k \in \mathscr{K}}(k(f))_{1}(x), \inf _{k \in \mathscr{K}}(k(f))_{2}(x)\right)
$$

whenever $x \in X$. Since $k(f) \in \mathscr{P}$, we have that $s(f) \in \mathscr{P}$.

Indeed observe that $s(f) \leq k(f) \leq f$, for all $f \in u P_{X}$. Thus $s(f) \leq f$ and condition (a) is satisfied.

To check condition (b), we check that $N(s(f), s(g)) \leq$ $N(k(f), k(g)) \leq N(f, g)$.
Indeed

$$
\begin{aligned}
N(f, g) \geq N(k(f), k(g)), \quad \text { since } k \in \mathscr{P} \\
\geq \sup _{x \in X}\left\{n\left((k(f))_{1}(x),(k(g))_{1}(x)\right)\right\} \\
\quad \vee \sup _{x \in X}\left\{n\left((k(g))_{2}(x),(k(f))_{2}(x)\right)\right\} \\
\geq \sup _{x \in X} \inf _{k \in \mathscr{K}}\left\{n\left((k(f))_{1}(x),(k(g))_{1}(x)\right)\right\} \\
\quad \vee \sup _{x \in X} \inf _{k \in \mathscr{K}}\left\{n\left((k(g))_{2}(x),(k(f))_{2}(x)\right)\right\} \\
\geq \sup _{x \in X} n\left(\inf _{k \in \mathscr{K}}(k(f))_{1}(x), \inf _{k \in \mathscr{K}}(k(g))_{1}(x)\right) \\
\quad \vee \sup _{x \in X} n\left(\inf _{k \in \mathscr{K}}(k(g))_{2}(x), \inf _{k \in \mathscr{K}}(k(f))_{2}(x)\right) \\
=\quad N(s(f), s(g)) .
\end{aligned}
$$

Thus we have that condition (b) is satisfied and since $s$ is a map from $u P_{X}$ to $u P_{X}$, we conclude that $s \in \mathscr{P}$ and $s$ is a lower bound of the chain $\mathscr{K}$ by construction. We therefore appeal to Zorn's lemma to conclude that $\mathscr{P}$ has a minimal element, say $m$, with respect to the partial order $\preceq$.

To complete the proof, we show that $m(f) \in u Q_{X}$ whenever $f \in u P_{X}$.

For each $x \in X$, we have that $p_{x} \circ m \in \mathscr{P}$ and $p_{x} \circ m \preceq m$ (where $p_{x}$ is as defined in the proof of Proposition 15). Thus by minimality of $m$, we have $p_{x} \circ m=m$. It therefore follows that, for each $x \in X, p_{x}(m(f))=m(f)$ whenever $f \in u P_{X}$. Thus by the way elements in $u Q_{X}$ are defined, we conclude that $m(f) \in u Q_{X}$ whenever $f \in u P_{X}$.

Proposition 18 (compare [1, Proposition 3]). Let $(Y, d)$ be $U Q P$-metric space and let $\emptyset \neq X$ be a subspace of $(Y, d)$. Then there exists an isometric embedding $\tau: u Q_{X} \rightarrow u Q_{Y}$ such that $\left.\tau(f)\right|_{X}=f$ whenever $f \in u Q_{X}$.

Proof. Fix $x_{0} \in X$ and let $p: u P_{Y} \rightarrow u Q_{Y}$ be a retraction satisfying the conditions of Proposition 17. Also let $s$ : $u Q_{X} \rightarrow u P_{Y}$ be such that $s(f)=f^{\prime}$, where $f_{1}^{\prime}(y)=$ $f_{1}(y)$ whenever $y \in X$, and $f_{1}^{\prime}(y)=\max \left\{f_{1}\left(x_{0}\right), d\left(x_{0}, y\right)\right\}$ whenever $y \in Y \backslash X$. The coordinate $f_{2}^{\prime}$ of pair $f^{\prime}$ is defined similarly.

We will consider the following cases to prove that $f^{\prime}$ belongs to $u P_{Y}$.

Case 1. Consider that $x \in X$ and $y \in X$.

Then $\max \left\{f_{2}^{\prime}(x), f_{1}^{\prime}(y)\right\}=\max \left\{f_{2}(x), f_{1}(y)\right\} \geq d(x, y)$.

Case 2. One has that $x \in Y \backslash X$ and $y \in Y \backslash X$.

Then

$$
\begin{aligned}
& \max \left\{f_{2}^{\prime}(x), f_{1}^{\prime}(y)\right\} \\
& =\max \left\{f_{2}\left(x_{0}\right), f_{1}\left(x_{0}\right), d\left(x, x_{0}\right), d\left(x_{0}, y\right)\right\} \\
& \geq \max \left\{d\left(x, x_{0}\right), d\left(x_{0}, y\right)\right\} \geq d(x, y) .
\end{aligned}
$$


Case 3. Consider that $x \in X$ and $y \in Y \backslash X$.

Then $\max \left\{f_{2}^{\prime}(x), f_{1}^{\prime}(y)\right\}=\max \left\{f_{2}(x), f_{1}\left(x_{0}\right), d\left(x_{0}, y\right)\right\} \geq$ $\max \left\{d\left(x, x_{0}\right), d\left(x_{0}, y\right)\right\} \geq d(x, y)$.

Case 4. Consider that $x \in Y \backslash X$ and $y \in X$.

Then $\max \left\{f_{2}^{\prime}(x), f_{1}^{\prime}(y)\right\}=\max \left\{f_{2}\left(x_{0}\right), f_{1}(y), d\left(x, x_{0}\right)\right\} \geq$ $\max \left\{d\left(x, x_{0}\right), d\left(x_{0}, y\right)\right\} \geq d(x, y)$.

Thus $f^{\prime} \in u P_{Y}$.

Define map $\tau=p \circ s$. Then $\left.\tau(f)\right|_{X}=\left.p\left(f^{\prime}\right)\right|_{X}=f$ whenever $f \in u Q_{X}$ since $p\left(f^{\prime}\right) \leq f^{\prime}$. Thus $\left.p\left(f^{\prime}\right)\right|_{X} \leq\left. f^{\prime}\right|_{X}=$ $f$, and $f$ is minimal on $X$.

Moreover for any $f, g \in u Q_{X}$, we have

$$
\begin{aligned}
N(f, g) & =N\left(\left.\tau(f)\right|_{X},\left.\tau(g)\right|_{X}\right) \leq N(\tau(f), \tau(g)) \\
& =N\left(p\left(f^{\prime}\right), p\left(g^{\prime}\right)\right) \leq N\left(f^{\prime}, g^{\prime}\right) \\
& =N(f, g) .
\end{aligned}
$$

The last equality follows from the definition of $f^{\prime}$ and $g^{\prime}$. Hence we have that $N(f, g) \leq N(\tau(f), \tau(g)) \leq N(f, g)$ which implies that $N(\tau(f), \tau(g))=N(f, g)$ and hence $\tau$ is an isometric map.

Definition 19. Let $X$ be a subspace of UQP-metric space $\left(Y, d_{Y}\right)$. Then $\left(Y, d_{Y}\right)$ is called $u q$-tight extension of $X$ if for any $u q p$-metric $\rho$ on $Y$ that satisfies $\rho \leq d_{Y}$ and agrees with $d_{Y}$ on $X \times X$, we have that $\rho=d_{Y}$.

Remark 20. For any UQP-metric uq-tight extension $Y_{1}$ of $X$, any $U Q P$-metric extension $\left(Y_{2}, d\right)$ of $X$ and any nonexpansive map $\varphi: Y_{1} \rightarrow Y_{2}$ satisfying $\varphi(x)=x$ whenever $x \in X$ must necessarily be an isometric map.

Indeed if that is not the case, then the uqp-metric $\rho: Y_{1} \times$ $Y_{1} \rightarrow[0, \infty)$ defined by $(x, y) \mapsto \rho(x, y)=d(\varphi(x), \varphi(y))$ would contradict the fact that the span $Y_{1}$ of $X$ is ultra-ample.

It was shown in [4] that the map $e_{X}:(X, d) \rightarrow\left(u Q_{X}, N\right)$ from $U Q P$-metric space $(X, d)$ to its ultra-quasi-metrically injective hull $\left(u Q_{X}, N\right)$ defined by $e_{X}(a)=f_{a}$ whenever $a \in X$ is an isometric embedding. We will proceed now with the help of Lemma 16 to show that $u Q_{X}$ is $u q$-tight extension of $e_{X}(X)$.

Proposition 21. Let $(X, d)$ be UQP-metric space and let $e_{X}$ : $X \rightarrow u Q_{X}$ be as defined above. Then $u Q_{X}$ is uq-tight extension of $e_{X}(X)$.

Proof. Let $\rho$ be uqp-metric on $u Q_{X}$ such that $\rho \leq N$ and $\rho\left(f_{x}, f_{y}\right)=N\left(f_{x}, f_{y}\right)$ whenever $x, y \in X$. By Lemma 16 and the fact that $\rho \leq N$, for any $f, g \in u Q_{X}$, we have

$$
\begin{aligned}
& N(f, g)=\sup _{x_{1}, x_{2} \in X}\left\{N\left(f_{x_{1}}, f_{x_{2}}\right): N\left(f_{x_{1}}, f_{x_{2}}\right)\right. \\
& \left.\quad>N\left(f_{x_{1}}, f\right), N\left(g, f_{x_{2}}\right)\right\} \\
& \quad \leq \sup _{x_{1}, x_{2} \in X}\left\{\rho\left(f_{x_{1}}, f_{x_{2}}\right): \rho\left(f_{x_{1}}, f_{x_{2}}\right)\right. \\
& \left.\quad>\rho\left(f_{x_{1}}, f\right), \rho\left(g, f_{x_{2}}\right)\right\} \leq \rho(f, g), \\
& \text { since } \rho\left(f_{x_{1}}, f_{x_{2}}\right) \leq \max \left\{\rho\left(f_{x_{1}}, f\right), \rho(f, g), \rho\left(g, f_{x_{2}}\right)\right\} .
\end{aligned}
$$

Thus $\rho=N$.
Proposition 22. Let $(Y, d)$ be UQP-metric uq-tight extension of $X$. Then the restriction map defined by $\left.f \mapsto f\right|_{X}$ whenever $f \in u Q_{Y}$ is a bijective isometric map $u Q_{Y} \rightarrow u Q_{X}$.

Proof. Let $p: u P_{X} \rightarrow u Q_{X}$ be a retraction map that satisfies the conditions of Proposition 17 and let $\varphi: u Q_{Y} \rightarrow u Q_{X}$ : $f \mapsto p\left(\left.f\right|_{X}\right)$ denote the composition of the retraction map $p$ with the restriction map. Then one sees immediately that $\varphi$ is nonexpansive. Thus by Lemma 3, $\varphi$ must be an isometry, because $u Q_{Y}$ is $u q$-tight extension of $X$ (this is so since $u Q_{Y}$ is $u q$-tight extension of $Y$ and $Y$ is $u q$-tight extension of $X$ ).

Choose $\tau: u Q_{X} \rightarrow u Q_{Y}$ an isometric embedding such that $\left.\tau(f)\right|_{X}=f$ for every $f \in u Q_{X}$ (compare Proposition 18). We therefore have

$$
\begin{aligned}
\varphi(\tau(f))=p\left(\left.\tau(f)\right|_{X}\right)=p(f) & f \\
& \text { for every } f \in u Q_{X} .
\end{aligned}
$$

This implies that $\varphi$ is onto. The fact that $\varphi$ is injective is clear since $\left(u Q_{X}, N\right)$ is $U Q P$-metric space (see, e.g., the last line of Definition 7). Thus $\varphi$ is bijective. In this case, the inverse of $\varphi$ has to be the inverse of $\tau$ and hence for any $f \in u Q_{Y}$, we have $\left.f\right|_{X}=\left.\tau(\varphi(f))\right|_{X}=\varphi(f) \in u Q_{X}$, which is the map

$$
u Q_{Y} \longrightarrow u P_{X}:\left.f \longmapsto f\right|_{X}
$$

that maps $u Q_{Y}$ onto $u Q_{X}$, without it being composed of $p$. Hence for any $U Q P$-metric $u q$-tight extension $Y$ of $X$, the map

$$
u Q_{Y} \longrightarrow u Q_{X}:\left.f \longmapsto f\right|_{X}
$$

is a bijective isometry between $u Q_{X}$ and $u Q_{Y}$.

Theorem 23. Let $X$ be a subspace of the UQP-metric space $(Y, d)$. Then the following are equivalent:

(a) $Y$ is UQP-metric uq-tight extension of $X$.

(b) $d\left(y_{1}, y_{2}\right)=\sup \left\{d\left(x_{1}, x_{2}\right): x_{1}, x_{2} \in X, d\left(x_{1}, x_{2}\right)>\right.$ $\left.d\left(x_{1}, y_{1}\right), d\left(x_{1}, x_{2}\right)>d\left(y_{2}, x_{2}\right)\right\}$ whenever $y_{1}, y_{2} \in Y$.

(c) $\left.f_{y}\right|_{X}(x)=(d(y, x), d(x, y)), x \in X$, is minimal on $X$ whenever $y \in Y$ and the map $(Y, d) \rightarrow\left(u Q_{X}, N\right)$ defined by $\left.y \mapsto f_{y}\right|_{X}$ is an isometric embedding.

Proof.

(a) $\Rightarrow(b)$. Let $Y$ be UQP-metric uq-tight extension of $X$. By Proposition 22, the restriction map $u Q_{Y} \rightarrow u Q_{X}$ is a bijective isometry between $u Q_{Y}$ and $u Q_{X}$. Thus the extension $Y \subseteq u Q_{Y}$ satisfies condition (b), since $u Q_{X}$ satisfies it by Lemma 16.

(b) $\Rightarrow(c)$. Let $x_{1}, x_{2} \in X$ and $y_{1} \in Y$. Then we have that $d\left(x_{1}, x_{2}\right) \leq \max \left\{d\left(x_{1}, y_{1}\right), d\left(y_{1}, x_{2}\right)\right\}$. Thus by condition (b) we have that $d\left(x_{1}, x_{2}\right) \leq d\left(y_{1}, x_{2}\right)$. Also

$$
d\left(x_{1}, x_{2}\right) \leq n\left(d\left(y_{1}, x_{2}\right), d\left(y_{2}, x_{2}\right)\right) \leq d\left(y_{1}, y_{2}\right) .
$$


Hence for $y_{1}, y_{2} \in Y$ we have by condition (b) that

$$
\begin{aligned}
& d\left(y_{1}, y_{2}\right)=\sup \left\{d\left(x_{1}, x_{2}\right): x_{1}, x_{2} \in X, d\left(x_{1}, x_{2}\right)\right. \\
& \left.\quad>d\left(x_{1}, y_{1}\right), d\left(x_{1}, x_{2}\right)>d\left(y_{2}, x_{2}\right)\right\} \\
& \quad \leq \sup \left\{d\left(y_{1}, x_{2}\right): x_{2} \in X, d\left(y_{1}, x_{2}\right)>d\left(y_{2}, x_{2}\right)\right\} \\
& \quad \leq d\left(y_{1}, y_{2}\right) .
\end{aligned}
$$

Similarly we have that $d\left(x_{1}, x_{2}\right) \leq \max \left\{d\left(x_{1}, y_{2}\right), d\left(y_{2}, x_{2}\right)\right\}$ whenever $x_{1}, x_{2} \in X$ and $y_{2} \in Y$ so that by condition (b) we get $d\left(x_{1}, x_{2}\right) \leq d\left(x_{1}, y_{2}\right)$. It therefore follows that, for each $x_{1}, x_{2} \in X$ and $y_{1}, y_{2} \in Y, d\left(x_{1}, x_{2}\right) \leq d\left(x_{1}, y_{2}\right)$. Thus for $y_{1}, y_{2} \in Y$ we see by (b) that

$$
\begin{aligned}
& d\left(y_{1}, y_{2}\right)=\sup \left\{d\left(x_{1}, x_{2}\right): x_{1}, x_{2} \in X, d\left(x_{1}, x_{2}\right)\right. \\
& \left.\quad>d\left(x_{1}, y_{1}\right), d\left(x_{1}, x_{2}\right)>d\left(y_{2}, x_{2}\right)\right\} \\
& \quad \leq \sup \left\{d\left(x_{1}, y_{2}\right): x_{1} \in X, d\left(x_{1}, y_{2}\right)>d\left(x_{1}, y_{1}\right)\right\} \\
& \quad \leq d\left(y_{1}, y_{2}\right) .
\end{aligned}
$$

Thus we conclude that $d\left(y_{1}, y_{2}\right)=N\left(\left.f_{y_{1}}\right|_{X},\left.f_{y_{2}}\right|_{X}\right)$.

As we have above, for any $y_{1}, y_{2} \in Y$ we have that

$$
\begin{aligned}
& d\left(y_{1}, y_{2}\right) \\
& =\sup \left\{d\left(y_{1}, x_{2}\right): x_{2} \in X, d\left(y_{1}, x_{2}\right)>d\left(y_{2}, x_{2}\right)\right\} \\
& d\left(y_{1}, y_{2}\right) \\
& =\sup \left\{d\left(x_{1}, y_{2}\right): x_{1} \in X, d\left(x_{1}, y_{2}\right)>d\left(x_{1}, y_{1}\right)\right\}
\end{aligned}
$$

Observe that if we substitute $x_{1} \in X$ for $y_{1}$ in (24) and $x_{2} \in X$ for $y_{2}$ in (25) we obtain the following equations:

$$
\begin{aligned}
& \left(f_{y_{2}}\right)_{2}\left(x_{1}\right)=d\left(x_{1}, y_{2}\right) \\
& \quad=\sup \left\{d\left(x_{1}, x_{2}\right): x_{2} \in X, d\left(x_{1}, x_{2}\right)>d\left(y_{2}, x_{2}\right)\right\}
\end{aligned}
$$

whenever $y_{2} \in Y$ and $x_{1} \in X$ and

$$
\begin{aligned}
& \left(f_{y_{1}}\right)_{1}\left(x_{2}\right)=d\left(y_{1}, x_{2}\right) \\
& \quad=\sup \left\{d\left(x_{1}, x_{2}\right): x_{1} \in X, d\left(x_{1}, x_{2}\right)>d\left(x_{2}, y_{2}\right)\right\}
\end{aligned}
$$

whenever $y_{1} \in Y$ and $x_{2} \in X$. We have therefore that restriction $\left.f_{y}\right|_{X}$ is minimal on $X$ whenever $y \in Y$ (compare Lemma 12).

$(c) \Rightarrow(a)$. Let $\rho$ be $u q p$-metric on $Y$ such that $\rho\left(y_{1}, y_{2}\right) \leq$ $d\left(y_{1}, y_{2}\right)$ whenever $y_{1}, y_{2} \in Y$ and $\rho\left(x_{1}, x_{2}\right)=d\left(x_{1}, x_{2}\right)$ whenever $x_{1}, x_{2} \in X$. Then by part (c) and the fact that $\left.f_{y}\right|_{X}$ is minimal whenever $y \in X$, we have

$$
\begin{aligned}
& d\left(y_{1}, y_{2}\right)=N\left(\left.f_{y_{1}}\right|_{X},\left.f_{y_{2}}\right|_{X}\right) \\
& \quad=\sup \left\{d\left(y_{1}, x\right): x \in X, d\left(y_{1}, x\right)>d\left(y_{2}, x\right)\right\} \\
& \quad \cdot \operatorname{by}(24) \\
& \quad=\sup \left\{d\left(x, y_{2}\right): x \in X, d\left(x, y_{2}\right)>d\left(x, y_{1}\right)\right\}
\end{aligned}
$$

by $(25)$.
By substituting

$$
\begin{aligned}
& d\left(x_{1}, y_{2}\right) \\
& \quad=\sup \left\{d\left(x_{1}, x_{2}\right): x_{2} \in X, d\left(x_{1}, x_{2}\right)>d\left(y_{2}, x_{2}\right)\right\}
\end{aligned}
$$

into formula

$$
\begin{aligned}
& d\left(y_{1}, y_{2}\right) \\
& \quad=\sup \left\{d\left(x_{1}, y_{2}\right): x_{1} \in X, d\left(x_{1}, y_{2}\right)>d\left(x_{1}, y_{1}\right)\right\}
\end{aligned}
$$

we obtain

$$
\begin{aligned}
& d\left(y_{1}, y_{2}\right)=\sup \left\{d\left(x_{1}, y_{2}\right): x_{1} \in X, d\left(x_{1}, y_{2}\right)\right. \\
& \left.>d\left(x_{1}, y_{1}\right)\right\}=\sup \left\{d\left(x_{1}, x_{2}\right): x_{1}, x_{2}\right. \\
& \quad \in X, d\left(x_{1}, y_{2}\right)>d\left(x_{1}, y_{1}\right), d\left(x_{1}, x_{2}\right) \\
& \left.>d\left(y_{2}, x_{2}\right)\right\}=\sup \left\{\rho\left(x_{1}, x_{2}\right): x_{1}, x_{2}\right. \\
& \in X, \rho\left(x_{1}, y_{2}\right)>\rho\left(x_{1}, y_{1}\right), \rho\left(x_{1}, x_{2}\right) \\
& \left.>\rho\left(y_{2}, x_{2}\right)\right\} \leq \rho\left(y_{1}, y_{2}\right)
\end{aligned}
$$

whenever $y_{1}, y_{2} \in Y$. The last inequality holds by the light of the inequality

$$
\rho\left(x_{1}, x_{2}\right) \leq \max \left\{\rho\left(x_{1}, y_{1}\right), \rho\left(y_{1}, y_{2}\right), \rho\left(y_{2}, x_{2}\right)\right\}
$$

and the fact that $\rho\left(x_{1}, x_{2}\right)>\rho\left(x_{1}, y_{1}\right)$ and $\rho\left(x_{1}, x_{2}\right)>$ $\rho\left(y_{2}, x_{2}\right)$. Thus we have that $\rho\left(y_{1}, y_{2}\right)=d\left(y_{1}, y_{2}\right)$ whenever $y_{1}, y_{2} \in Y$ and hence (a) follows.

Remark 24. We see from Theorem 23 that there is only one isometric embedding $\varphi: Y \rightarrow u Q_{X}$ satisfying $\varphi(x)=f_{x}$ whenever $x \in X$, since for such an embedding we have

$$
\begin{aligned}
\left.\left(f_{y}\right)_{2}\right|_{X}(x) & =d(x, y)=N(\varphi(x), \varphi(y)) \\
& =N\left(f_{x}, \varphi(y)\right)=(\varphi(y))_{2}(x) ;
\end{aligned}
$$

therefore $\left.\left(f_{y}\right)_{2}\right|_{X}=(\varphi(y))_{2}$. Similarly, one can show that $\left.\left(f_{y}\right)_{1}\right|_{X}=(\varphi(y))_{1}$ whenever $y \in Y$.

Thus we see that the uq-tight extension $Y$ of $X$ can be understood as a subspace of extension $u Q_{X}$ of $X$. Hence $u Q_{X}$ is maximal among the $U Q P$-metric $u q$-tight extensions of $X$.

\section{Conflict of Interests}

The author declares that there is no conflict of interests regarding the publication of this paper.

\section{References}

[1] C. A. Agyingi, P. Haihambo, and H.-P. A. Künzi, "Tight extensions of $T_{0}$-quasi-metric spaces," Logic, Computation, and Hierarchies, pp. 9-22, 2014.

[2] A. W. Dress, "Trees, tight extensions of metric spaces, and the cohomological dimension of certain groups: a note on combinatorial properties of metric spaces," Advances in Mathematics, vol. 53, no. 3, pp. 321-402, 1984. 
[3] J. Diatta, "Approximating dissimilarities by quasi-ultrametrics," Discrete Mathematics, vol. 192, no. 1-3, pp. 81-86, 1998.

[4] H.-P. A. Künzi and O. O. Otafudu, "The ultra-quasi-metrically injective hull of a $T_{0}$-ultra-quasi-metric space," Applied Categorical Structures, vol. 21, no. 6, pp. 651-670, 2013.

[5] M. M. Bonsangue, F. Van Breugel, and J. J. M. M. Rutten, "Generalized ultrametric spaces: completion, topology, and powerdomains via the Yoneda embedding," CWI Reports AP/CS-R9560, 1995. 


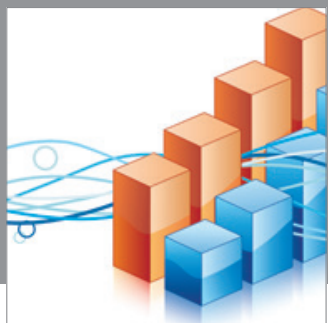

Advances in

Operations Research

mansans

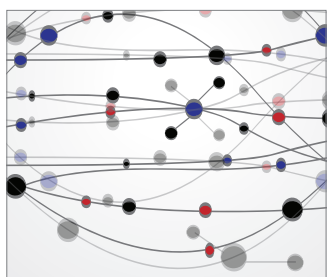

The Scientific World Journal
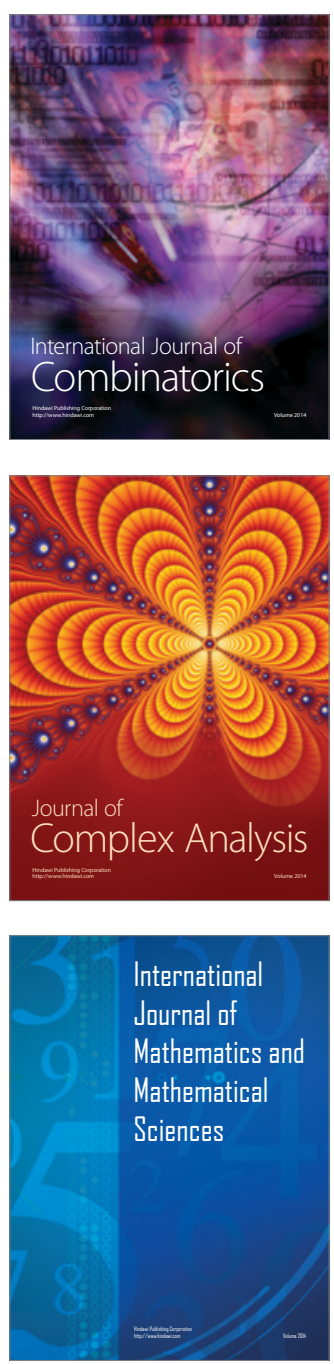
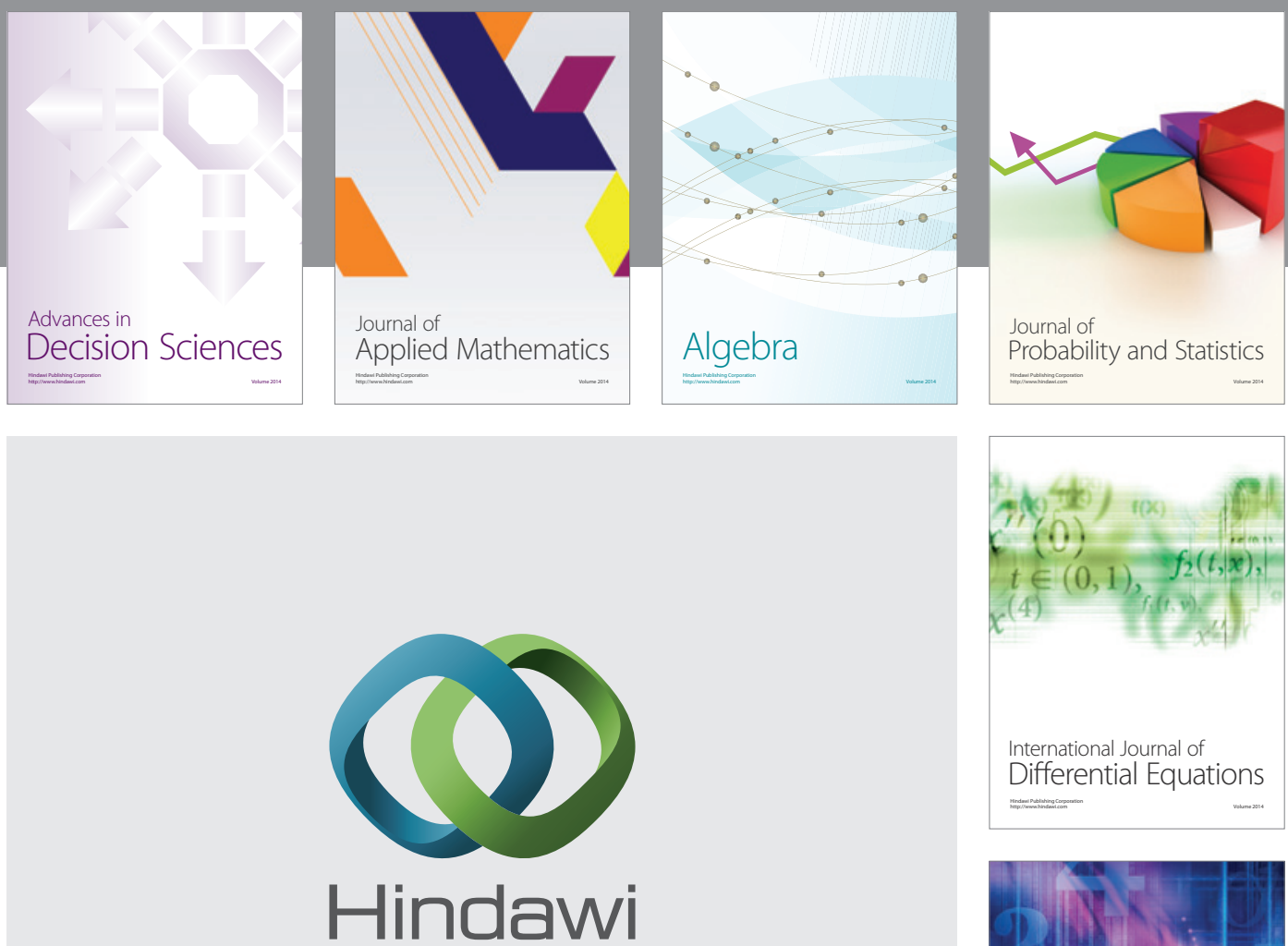

Submit your manuscripts at http://www.hindawi.com
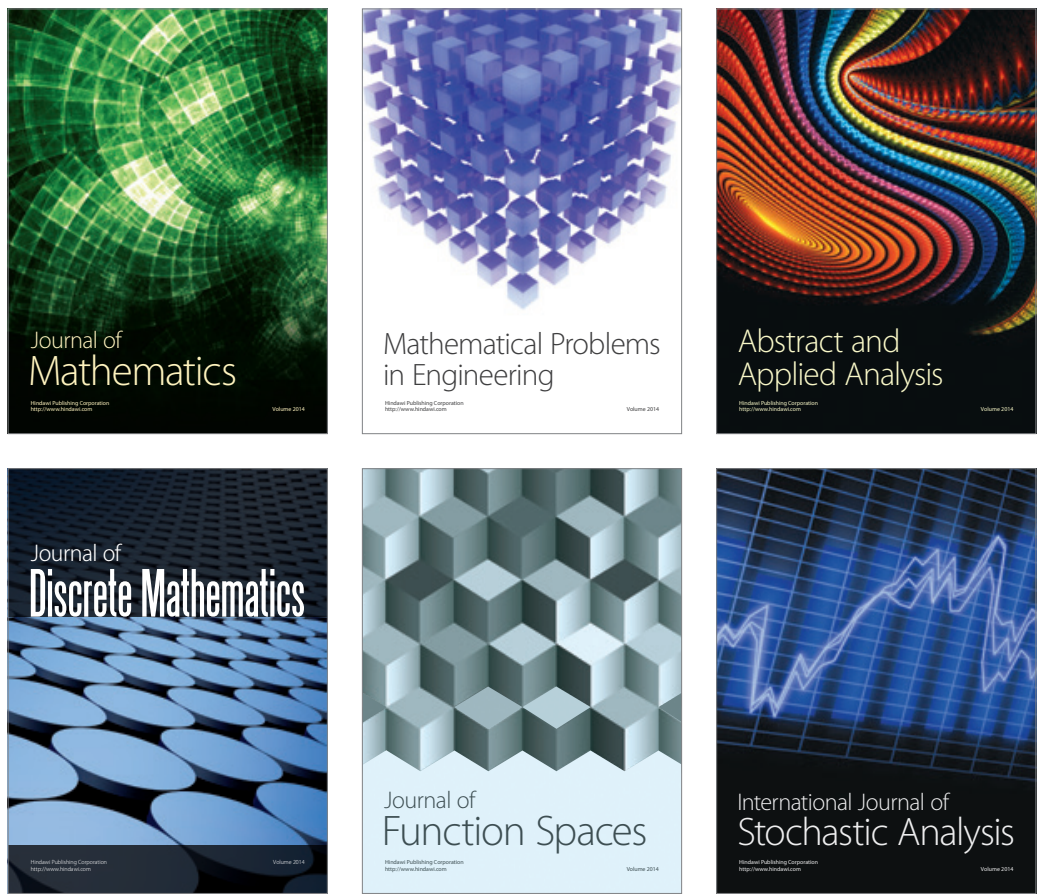

Journal of

Function Spaces

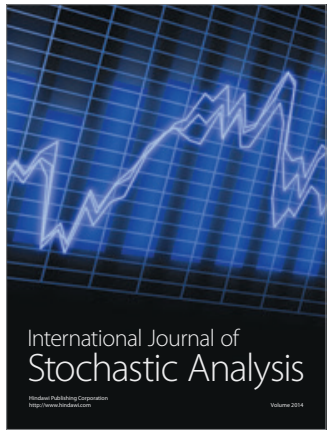

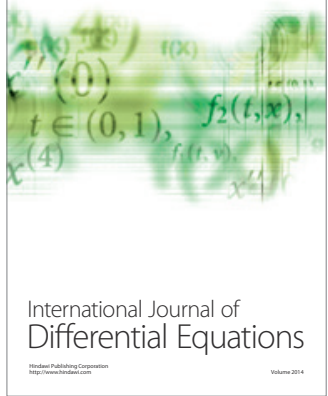
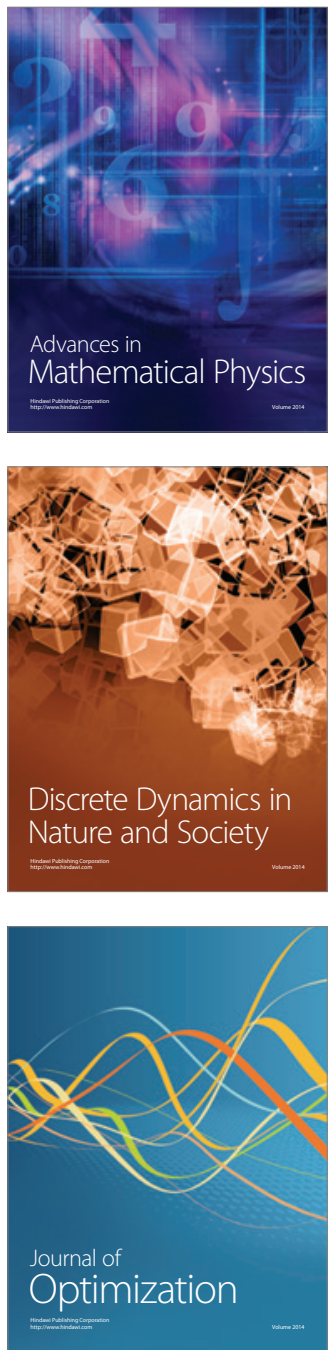